\title{
ANTI-FREE RADICALS: SOURCE, ROLE AND APPLICATION IN DERMO-COSMETOLOGY
}

\author{
Joëlle Joachim $^{1 *}$, G. Abdelbary ${ }^{1}$, P. Prinderre ${ }^{1}$, J.P. Reynier ${ }^{1}$, J. Kister ${ }^{2}$, P. Piccerelle ${ }^{1}$ \\ ${ }^{1}$ Laboratoire de Pharmacie Galénique et Pharmacotechnie industrielle, Biopharmacie et \\ Cosmétologie Faculté de Pharmacie- Université de la méditerranée 27 Bd Jean Moulin, \\ 13385 Marseille Cedex 5 \\ ${ }^{2}$ UMR CNRS 6171 Systèmes chimiques complexes, Faculté des Sciences de St Jérôme- GOAE \\ case 561 Université d'Aix-Marseille 3 Av Escadrille Normandie-Niemen, 13397 Marseille \\ cedex 20
}

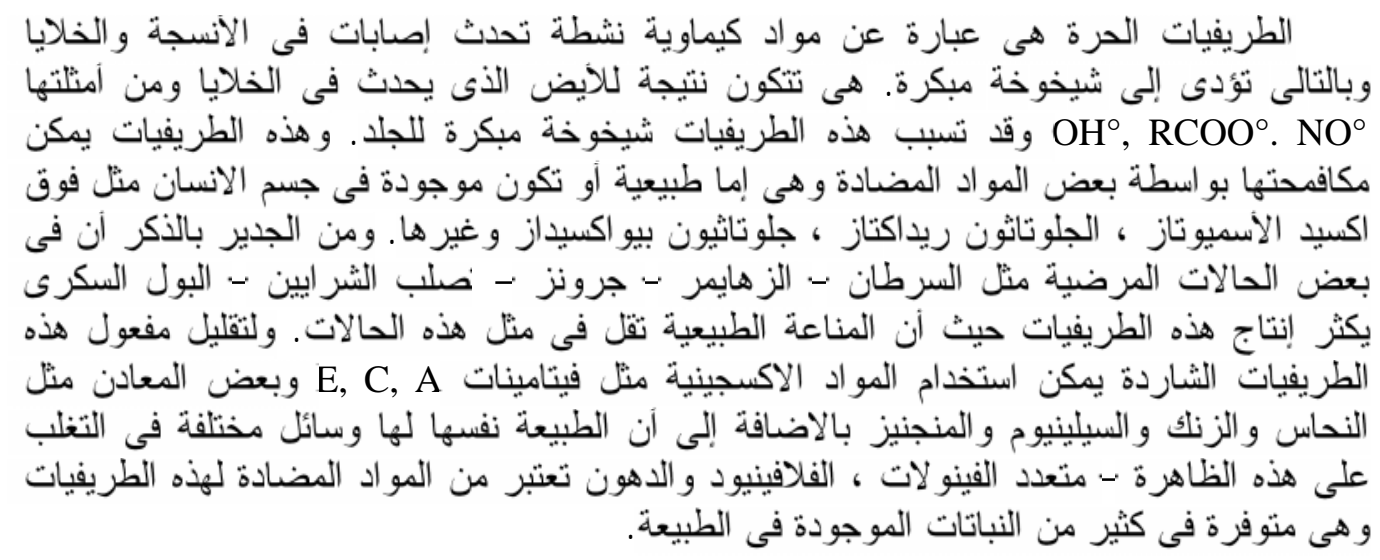

\begin{abstract}
Many environmental factors attack our skin continuously: Sun, wind, pollutants and tobacco which constitute the principle skin aggressors. Free radicals which are highly reactive chemical compounds, induce cellular and tissue injuries, and subsequently lead to aging. These free radicals are normally produced by cellular metabolism. They are represented by: $\mathrm{OH}^{\circ}$, $\mathrm{RCOO}^{\circ}, \mathrm{NO}^{\circ}$ radicals and singlet oxygen. Free radicals are involved in cutaneous aging. Normally these radicals are neutralised by natural or human antiradical agents like superoxide dismutase, glutathione reductase, glutathione peroxidase and others. Some pathologic situations (cancer, Alzheimer disease, Crohn's disease, atherosclerosis, diabetes mellitus ...) are characterized by an over production of these radicals where the natural defences become overwhelmed. Oral administration of exogenous substances like vitamin A, $C, E$, oligoelements $\mathrm{Cu}, \mathrm{Zn}, \mathrm{Se}, \mathrm{Mn}$ decrease free radical harmful and deleterious effects. Nature has several means to fight against oxidative stress: polyphenols, flavonö̈des and lipids are antiradical substances of plant origin. The future belongs to understand natural antiradical mechanisms as well as the synthesis of new molecules which would be effective in prevention of harmful effects produced by these radicals.
\end{abstract}

\section{INTRODUCTION}

We live a time where scientific terms like free radicals, anti-free radicals and others are frequently used. In fact, recent cosmetology is concerned with active principles and formulations that can embellish our skin and at the same time give it back its younger aspect.
What are these anti-free radicals that so much attract our attention? What is their role? Where can we find them? How can we evaluate their efficiency? In what type of dosage form and formulations can we incorporate them? To answer all these questions, it is important to recall what is a free radical and its implication in physio-pathological term.

Received in 28/2/2004 \& Accepted in 25/3/2004

*To whom correspondence should be addressed (E-Mail: joellejoachim@hotmail.com) 


\section{Free radicals}

\section{1- Definition}

The human cell needs energy in order to be able to function normally. This energy comes from breathed oxygen, which is essentially used by the mitochondria, that synthesizes ATP (Adenosin Tri-Phosphate) molecules. During this chain reaction, the poisonous molecules derived from the used oxygen escape. These very reactive molecules are called free radicals (FR). $\mathrm{FR}^{1}$ may be defined as an atom or a group of atoms presenting an unpaired electron in its outer orbital. These entities greedy for electrons are susceptible to react immediately with other molecular species where they alter their chemical structure and biologic properties. There are different types of FR derived from hydrogen peroxide or carboxylic acid. For example, one can mention the superoxide $\mathrm{O}_{2}{ }^{\circ}$ radical, the hydroxyl radical $\mathrm{OH}^{\circ}$, the singlet oxygen ${ }^{1} \mathrm{O}_{2}$, the alcoxy and peroxy radicals. These activated species of oxygen are differentiated by their reactivity, their half-life or by their affinity towards the cellular membranes. Besides the breathed oxygen, other exogenous factors ${ }^{2}$ are also responsible for production of FR, among those factors: tobacco smoke, pollutants, wind and sun. These environmental factors constitute the main aggressors of our skin.

\section{2- Pathological implication}

FR are implied in numerous phenomenon which may be physiological as the cutaneous aging or pathological $^{3}$ as cancer, atherosclerosis, cardio-vascular diseases, hypertension, arthritis, diabetes, neurodegenerative diseases as Parkinsonism, Alzheimer or inflammatory diseases of the digestive tract as hemorrhagic recto-colitis or Crohn's disease. In all these diseases, FR increase production of the inflammatory factors as PG, leucotrienes, and thromboxane. FR are responsible for cellular death exactly like inflammation or calcium excess. This article is interested in the phenomenon of cutaneous aging.

\section{3- Cellular defense mechanism}

The human cell is subjected to multiple aggressions. To defend itself against these attacks of all nature, it is endowed of repair mechanisms for molecular lesions and elimination of damaged elements. ${ }^{4}$ Enzymatic cellular defense is essentially composed of catalase, glutathion reductase, glutathion peroxidase and superoxide dismutase. The cell also possesses a battery of hydrosoluble or liposoluble components that participate in the resistance against oxidative stress. These antifree-radicals will be detailed further and some of them can be mentioned here:

- Vitamins and derivatives (pro-vitamin A, vitamin $\mathrm{C}$ and $\mathrm{E})$.

- Uric acid, glutathion, melatonin, methionin,

- Co-factor (Q coenzyme, selenium).

\section{Photo induced aging \\ 1- Definition of aging}

Four main characters define the aging process: there are intrinsic, irreversible, progressive, and universal process. ${ }^{1}$ The energetic, genetic and environmental factors influence the cutaneous aging. These factors affect maintenance, repair processes and cellular deterioration. Currently, one can affirm that the deleterious action of the FR contributes to the acceleration of the aging.

\section{2- Effects of Free radicals on the skin}

Among environmental factors that contribute to mark everyday the weight of years, one can note that solar radiance is responsible for the helio-dermis phenomenon commonly called photo induced aging. The solar exposures either exaggerated or too frequent are the origin of premature aging. However, it is important to mention that the quantity of solar radiance absorbed by the skin essentially varies according to time of exhibition, the reflection of the surface to soil, the latitude, the altitude and the hygrometric degree of air. ${ }^{5}$ The solar radiation impact is not only a function of exogenous factors as previously quoted but also of endogenous factors as the type of cutaneous pigmentation and the thickness of the stratum corneum. Among the solar radiation, only UV A and B radiations are of interest. One can recall that the $\mathrm{UV} A$ is melanogene and the $\mathrm{UV} \mathrm{B}$ is erythematic. The UV A radiations traverse the different layers of the skin (stratum corneum, layer of Malpighi and basal layer) to reach the papillary dermis, and so arrive at the target molecules. The photo induced aging causes innumerable visible cutaneous damages on the 
macroscopic level such as cutaneous deterioration, hyper-pigmentation, solar erythema, hyper-keratosis and particularly premature aging. On the microscopic level, there is molecular and tissue lesions, melanogenesis and endogenous antioxidant depletion of about $60 \%-70 \% .^{6}$ The solar radiations are beneficially responsible for urocanic acid production that transported by sweat to the skin surface, where it absorbs erythematic UV. Among the damages induced by UV radiations via FR on the tissue level, dermic lesions essentially appear by changes in collagen and elastic fibers. This is called solar elastosis which is characterized by elastic fiber thickening, aggregation and fragmentation. The degree of elastosis is proportional to the quantity of received radiations. These signs of cutaneous senility are also attributable to collagen fibers, which under the effect of radiations, are going to decrease in number thus decreasing the thickness of the dermis. They are also going to lose their springiness and thus give an important structural rigidity to the dermis.

On the molecular level, FR targets are the basic constituents of the human cell. The desoxyribonucleic acid (DNA), proteins and lipids are attacked by the UV radiations. An oxidative stress is going to generate, on the nucleic acid level, oxidation, reduction reactions, or combination of both bases (formation of thymidic dimeres). The FR also attack lipids that constitute peri-cellular and intracellular membranes causing a fluidity defect which can reach the complete lyses of the membrane via the phenomenon of lipoperoxidation. The solar radiations attack chromophore molecules of the skin as the urocanic acid, as they absorb solar radiations at wavelength $(\lambda)$ from 200 to $400 \mathrm{~nm}$ of the solar spectrum. This absorption of energy liberates FR that cause lipoperoxidation of unsaturated fatty acid of lysosomal membranes, provoking lyses of these membranes and liberation of hydrolases that they contained. Liberated hydrolases are responsible for cellular destruction and production of sun burn cells.

\section{Anti-free radicals \\ 1- Vitamins and oligo-elements: natural antioxidants \\ Vitamins are subdivided into two groups:}

- Hydrosoluble vitamins as the ascorbic acid or vitamin $C$ localized in the cytoplasm;

- Liposoluble vitamins as $\beta$ carotene or provitamin $\mathrm{A}$ and $\alpha$ tocopherol or vitamin $\mathrm{E}$.

Mainly Zinc, Copper, Manganese and Selenium represent anti-free radical oligoelements.

\section{Role}

Anti-free radicals act in different levels:

- Vitamin A inhibits lipid peroxidation and protects against the UV radiations;

- Vitamin $\mathrm{C}$ has an antioxidant action localized in the cytoplasm. It is an agent with double antioxidant actions. Does it have an intrinsic antioxidant action in aqueous phase it also capable of regenerating the vitamin $\mathrm{E}$ from its oxidized radical.

- Vitamin E, as the vitamin A, inhibits lipid peroxidation. It also corrects the deficit in selenium.

Alpha tocopherol appears like a predominant antioxidant at the level of stratum corneum. ${ }^{7}$ Vitamin $\mathrm{C}$ and $\mathrm{E}$ have protective effect against erythema, formation of thymine dimeres, and sun burn cells.

- Zinc, Copper and Manganese are co-factors of enzymatic cellular defense system.

- Selenium ${ }^{8}$ stops lipid oxidation, also acts as an immuno-stimulant agent and is mainly the active center of glutathion peroxidase.

\section{Sources}

The main sources of natura antioxidants ${ }^{9,10}$ are represented in the following table:

\begin{tabular}{||l|l||}
\hline $\begin{array}{c}\text { Antioxidant } \\
\text { s }\end{array}$ & \multicolumn{1}{c|}{ Sources } \\
\hline Vitamin A & Milk, butter, eggs \\
\hline Vitamin E & Litmus and germ wheat oil \\
\hline Vitamin C & $\begin{array}{l}\text { Citrus fruits, kiwi, green } \\
\text { cabbage }\end{array}$ \\
\hline Selenium & Wheat, fish, red meat, eggs \\
\hline
\end{tabular}

The pro-oxidizing effect of anti-free radicals

Antioxidants can be oxidized, generate some active substances and be considered as pro-oxidant substances. ${ }^{10,11}$ The pro-oxidizing effect of vitamin $\mathrm{C}$ appears in certain conditions according to its concentration, the level of catalytic metal that is in turn function 
of every skin type, and the nature of dosage form in which it is incorporated. In fact, a low concentration of vitamin $\mathrm{C}^{12}$ the level of catalytic ions $\left(\mathrm{Fe}^{3+}\right)$ in the skin and an emulsion type formulation «Oil in Water » are favourable of a pro-oxidant action. It is possible to add a metal chelator for example deferoxamine to palliate the pro-oxidant action of low concentration of vitamin $\mathrm{C}$.

\section{Other activities of antioxidants}

Antioxidant activity is not the only property of these agents. ${ }^{10}$ In fact, they interfere with bacterial, viral or fungal aggressions, and participate in defending against microorganism by their natural antibacterial property. These agents have antiinflammatory action against the multiple inflammatory diseases as Crohn's, Alzheimer. They also have anti-ulcer, immune-modulating, anti-carcinogenic and anti-metastatic activity.

\section{2- Antioxidants of synthetic origin}

Currently, numerous researchers are concerned by the synthesis of new antioxidant compounds. One can mention, the BHT (butyl hydroxy toluene), the BHA (butyl hydroxy anisol), lazaroïds, the PBN (phenyl $\alpha$ butylnitrone) and derivatives. It is worthy to note that these antioxidants of synthetic origin possess a superior anti-free radical action to that of natural antioxidants. Unlike natural antioxidants synthetic ones can not be widely used except in limited quantities by reason of their chemical nature.

\section{3- Antioxidants of plant origin}

There are more than 8000 active compounds of plant origin and among them; the most important are polyphenols and flavonoids.

Two main families illustrate the study of antioxidants of plant origin:

- Family Labiatae that is composed of mint, thyme, rosemary, sage....

- Family Oleaceae as the olive tree.

Some parts of the olive tree are rich in polyphenols ${ }^{11}$ and flavonoids ${ }^{13}$ whose activity is superior to the product of reference, as vitamin E, C and BHT. Polyphenols are also present in green tea and wine. They protect against deleterious free radical activity on the biologic molecules (DNA, lipids, proteins). Other antifree radical species are found in the 3 varieties of tea: green tea, oolang and black tea. Antioxidant activity is a function of the species. We can find phenolic derivatives for example, rosmarinic acid in sage, which inhibits peroxidation of lipids. The different constituents of sage can be extracted in aqueous phase or oily phase.

Silymarin extract of seeds, leaves and fruits containing flavonoids, used by oral or topical route, play a role in inhibition of peroxidation of lipids and formation of thymidic dimers. It also protects against the deleterious action of carcinogenic promoters as UV radiations. On the other hand, they extract from soy; isoflavons protect against the erythema, inflammation and change of the DNA caused by the PUVA therapy [the use of psoralene (P) followed by exposure to long wave ultraviolet light]. Some anti-free radical as flavoglycosids extracted from ginkgo biloba ${ }^{14}$ have an extensively superior activity to enzymatic system of defence as superoxide dismutase.

The plant kingdom is an immense source; besides their activity of free radical scavengers, some plants have detoxification properties as grapes or anti-carcinogenic properties as curcuma and ginger.

\section{4- Formulations, dosage forms and route of administration}

From medical point of view, antioxidants are very widly used in preventive treatment for sub carence of kids or defaulting of aging subjects. In curative treatment to re-establish the balance « oxidant - antioxidant » unsettled in certain pathologies (cancer, cardiovascular affections, chronic inflammation). From dermo-cosmetic point of view, numerous specialties are commercialized. The route of administration can be oral, called nutri-therapy or topical in the form of solutions, emulsions, or suspensions. As for the oral route, we can mention the Radicopen ${ }^{15}$ available in the form of tablets, composed of Vitamin C, Vitamin E, tomato extract, grape extract, Selenium, and olive oil. For the topical route, a lotion called serum $\mathrm{C}$ is composed of pine peels, bay of juniper, rosemary, tea and grape is available.

Lately, transdermic systems are marketed as patches $^{16}$ that provide a progressive liberation of the active principle. This dosage form is innovative because it permits prolonged release in situ of active principles. 


\section{5- Assessment of the antioxidants activity}

There are several methods that enable the measurement of the anti-free radical activity. ${ }^{10}$ The widly used is the measurement of the total DNA destroyed by the oxidative damage. The following methods are used in the measurement of:

- Antioxidant enzyme level

- Antioxidants of weak molecular weight level

- Lipid oxidative damage

- Protein oxidative damage

- Oxidative deterioration

- Inhibition of LDL (Low Density Lipoprotein) oxidization

These methods are based on the quantification of either the antioxidant activity, or damages caused by the free radicals.

\section{Test of resistance to free radical}

This test is a biologic test that measures the global anti-free radical defence power. Considering the totality and diversity of compounds (vitamins, anti-oxidants, prooxidizing agents), of enzymatic systems (glutathion peroxidase, catalase, superoxide dismutase) interfering in the anti-free radical defense system. All specific tests can not give a global appreciation of the resistance capacity of an individual against free radical aggression.

\section{Principle of the test}

It consists of submitting red globules to an oxidative aggression in strictly controlled and standardized conditions. In these conditions, hematite uses all their enzymatic and molecular assets to resist this aggression until the cellular membrane is modified and finally let escape its cellular content. The resistance of the tested red globules population is therefore expressed by the time for the liberation of $50 \%$ of the hemoglobin content. The medical interest of this test is to reflect, in a global way, the defense potential of an individual against free radical aggression implied in numerous pathologies and that it takes in consideration all positive factors (vitamins, enzymatic defense...) or negative factors (tobacco, alcoholism, stress, unbalanced nutrition, pollution, etc. ...). This test permits to follow the positive or negative evolution of a prescription, on the defense state of the individual against free radicals. Besides, it has been used to follow-up anti-free radical power of pharmaceutical molecules in vitro and in vivo permitting the observation of the possible negative effect related to a metabolite, even though the incriminated metabolite is not yet identified. Therefore this test considers the genetic heritage of an individual and his antifree radical defense, of his life mode, of known or unknown aggression situations. Thus this test may be used in preventive medicine or in therapeutic follow-up.

\section{Conclusion}

Free radicals can be naturally produced during cellular metabolism, or generated by several environmental factors, or they may be also implicated in numerous pathologies. Human cells possess many defence mechanisms, which could be either enzymatic like SOD (superoxide dismutase) or molecular like Vitamin A, C, and E. Both types fight against the hazardous effects that are caused by reactive species of oxygen. In some pathological and physiological cases such as aging, cellular defence is overwhelmed and consequently human cells become the target of free radicals which attack its fundamental components: proteins, lipids and nucleic acids.

The discovery of antioxidants of plant origin or their synthesis represents a great hope in the treatment of various pathologies where free radicals are implicated. In addition, certain antioxidants of plant origin have other useful and interesting activities like anti-inflammatory and anti-carcinogenic effects. Cutaneous aging which is an physiological phenomenon will encounter many changes in its evolution that would be owed to the new research concerning anti-free radicals.

\section{REFERENCES}

1- Guy Jadot. Antioxidants et vieillissement. John Libbey Eurotext, Paris (1994).

2- Internet site http://www.parisbeauté.fr. Radicaux libres et antioxydants, accessed on October (2003).

3- Internet site http://www.lecommuniquesante. ch. Les bienfaits des antioxydants. accessed on October (2003).

4- Internet site http://www.preventaging.com. Centre de Prévention du Vieillissement, accessed on October (2003). 
5- G. Ramette. Le Check-up de la Peau. Arnette, Manuel Pratique de médecine esthétique, Paris (1990).

6- M. Podda, M. G. Traber, C. Weber and L.J. Yan, L. Packer, Free Radical Biology \& Medicine, 24, 55 (1998).

7- J. J. Thiele, Oxidative Stress in the Skin Barrier: Implications for Stratum Corneum Antioxidants. Heinrich-Heine-University, Duesseldorf, Germany.

8- H. Tapiero, D. M. Towsend and K. D. Tew, Biomed. \& Pharmacotherapy, 57, 134 (2003).

9- J-P Goussard. Les Radicaux Libres et Antioxydants. D. Riche in guide nutritionnel des sports d'endurance, 1999, pp. 2-11.

10- A. Moure, J. M. Cruz, D. Franco, J. M. Dominguez, J. Sineiro, H. Dominguez, M. J. Nuñez and J. C. Parajo, Food Chemistry, 72, 145 (2001).
11- B. A. Jurkiewicz and S. P. Granger. The Prooxidant and Antioxidant Effects of Ascorbate Supplementation of Skin Keratinocytes. Unilever Research, 45 River Road, Edgewater, NJ 07020 USA.

12- P. Jurkovic, M. Sentjurc, M.Gasperlin, J. Kristl and S. Pecar, Europ. J. Pharm. Biopharma, 56, 59 (2003).

13- R. Aquino, S. Morelli, A. Tomaino, M. Pellegrino, A. Saija, L. Grumetto, C.Puglia, D. Ventura and F. Bonina, J. Ethnopharmacology, 79, 183 (2002).

14- Internet site http://www.mdidea.com. Ginkgo Biloba, accessed on October (2003).

15- Internet site http://www.lycored.com. Complément nutritionnel antiradicalaire, accessed on October (2003).

16- Internet site http://www.lanaform.com Vita Patch, accessed on October (2003). 